\title{
Passive Localization for Mixed Near-Field and Far-Field Sources Based on Cumulant Sparse Representation
}

\author{
Qinghua Liu, Wei Chen and Liangnian Jin \\ Guangxi Experiment Center of Information Science \\ Guilin University of Electronic Technology, Guilin, 541004, China \\ winner_chenwei@163.com
}

\begin{abstract}
In this paper, a new mixed near-field and far-field sources localization method based on cumulant sparse representation was proposed. By constructing a special cumulant matrix which is only related to the DOA parameters of mixed sources, we obtain the DOA estimation of all sources using the weighted $\ell_{1}$-norm algorithm. And then, a mixed overcomplete matrix on the basis of DOA estimation is introduced in the sparse signal representation framework to estimate the range parameters and distinguish far-field sources from the mixed sources. The proposed algorithm avoids two-dimensional searching and parameter matching, moreover, it has higher resolution and better accuracy. Simulation results verify the effectiveness of the proposed algorithm.
\end{abstract}

Keywords: Fourth-order cumulant; Sparse representation; Near-field; Far-field; DOA estimation

\section{Introduction}

Source localization is a key problem in array signal processing and has received significant attention in the last several decades. For far-field sources, large amount of algorithms have been proposed to achieve the direction-of-arrival (DOA) estimation, among them the ESPRIT algorithm [1] and MUSIC algorithm [2] are most representative. When a source lies in the near-field region, the assumption of plane waves for far-field sources no longer holds true. In this case, the wavefront must be characterized by not only the azimuth DOA, but also range parameter. Numerous effective methods, such as the two-dimensional MUSIC method [3] and the high-order ESPRIT method [4], are also available to deal with this issue.

However, in some practical applications, the signals received by the array are often the mixture of near-field and far-field sources. Liang [5] proposed a high-order MUSIC algorithm to estimate the parameters of the mixed sources, one weakness of this algorithm is that it suffers high computation complexity. In order to reduce the computation complexity, a second-order statistic (SOS)-based algorithm was suggested in [6], but it needs to know the number of near-field sources in advance. A second-order MUSIC algorithm was presented in [7], it has a great aperture loss which in turn makes it suffer serious performance degradation. In [8], by utilizing sparse signal reconstruction for mixed source localization to improve estimation accuracy and resolution, a weakness of it is undemocratic penalization for large coefficients incurs the degradation of signal recovery performance. To conquer this problem, the weighted $\ell_{1}$-norm minimization [9] was proposed, but the computational complexity influenced by the number of sensors seriously.

In order to overcome the aforementioned shortcoming of the mixed sources localization, we proposed a new mixed source localization algorithm based on cumulant sparse signal recovery. In this paper, Firstly, we use the fourth-order cumulant matrix to 
eliminate the range parameter of near-field sources, all the azimuth of incoming signals can be estimated by weighted $\ell_{1}-$ norm algorithm. Then, another special cumulant matrix which contains the DOAs and ranges parameter is constructed to estimate range parameters and distinguish far-field sources from the mixed sources. Simulation results are provided to demonstrate the performance improvement of the proposed method.

\section{Mixed Near-Field and Far-Field Signal Model}

Suppose that $K$ narrow-band, independent mix sources impinging on a uniform linear array with $2 M+1$ sensors as [10]. The center sensor regard as phase reference point. Data of the $m$ th sensor received can be expressed as

$$
x_{m}(t)=\sum_{k=1}^{K} s_{k}(t) \mathrm{e}^{j \tau_{m k}}+n_{m}(t), \quad t=0, \cdots, T-1
$$

where $T$ is the snapshot number, $s_{k}(t)$ is the $k$ th narrow-band source signal, $n_{m}(t)$ is the additive Gaussian noise, $\tau_{m k}$ is the delay associated with the $k$ th source propagation time between the 0 th and $m$ th sensor. When the source located at near-field, $\tau_{m k}$ can be given by

$$
\tau_{m k}=m \mu_{k}+m^{2} \phi_{k}
$$

where $\mu_{k}$ and $\phi_{k}$ can be expressed as

$$
\begin{aligned}
& \mu_{k}=-2 \pi \frac{d}{\lambda} \sin \left(\theta_{k}\right) \\
& \phi_{k}=\pi \frac{d^{2}}{\lambda r_{k}} \cos ^{2}\left(\theta_{k}\right)
\end{aligned}
$$

where $\lambda$ is the wavelength, $\theta_{k}$ and $r_{k}$ are the $k$ th source azimuth and range parameter. When the source located at far-field, $\phi_{k}$ can be considered as zero, $\tau_{m k}$ can be expressed as

$$
\tau_{m k}=m \mu_{k} .
$$

The received data can be expressed as a matrix form

$$
\mathbf{x}(t)=\mathbf{A s}(t)+\mathbf{n}(t)
$$

where

$$
\begin{aligned}
\mathbf{x}(t) & =\left[x_{-M}(t), \cdots, x_{0}(t), x_{1}(t), \cdots, x_{M}(t)\right]^{T} \\
\mathbf{A} & =\left[\mathbf{a}\left(\mu_{1}, \phi_{1}\right), \cdots, \mathbf{a}\left(\mu_{k}, \phi_{k}\right), \cdots, \mathbf{a}\left(\mu_{K}, \phi_{K}\right)\right] \\
\mathbf{s}(t)= & {\left[s_{1}(t), \cdots, s_{k}(t), \cdots s_{K}(t)\right]^{T} } \\
\mathbf{n}(t) & =\left[n_{-M}(t), \cdots, n_{0}(t), n_{1}(t), \cdots, n_{M}(t)\right]^{T} .
\end{aligned}
$$

If the source lies in near-field, the steering vector can be written as 


$$
\mathbf{a}\left(\mu_{k}, \phi_{k}\right)=\left[e^{j\left[(-M) \mu_{k}+(-M)^{2} \phi_{k}\right]}, e^{j\left[(-M+1) \mu_{k}+(-M+1)^{2} \phi_{k}\right]}, \cdots, e^{j\left[M \mu_{k}+M^{2} \phi_{k}\right]}\right]^{T}
$$

Otherwise, if the source located at far-field, the array manifold vector can be expressed as

$$
\mathbf{a}\left(\mu_{k}\right)=\left[e^{j\left[(-M) \mu_{k}\right]}, e^{j[(-M+1)] \mu_{k}}, \cdots, e^{j\left[(M) \mu_{k}\right]}\right]^{T} .
$$

In this paper, the following hypotheses are required:

1. The signals are statistically independent, zero mean stationary process.

2. The noise is zero-mean, circular Gaussian, spatially uniform white and independent from the source signals.

3. The sensor array is a symmetric uniform line array. To avoid an ambiguity of phase in mixed sources localization scenario, the inter-element spacing is within a quarter-wavelength.

\section{Proposed Method}

\subsection{DOA Estimation}

In this section, a cumulant is considered to eliminate the Gaussian noise and to improve the accuracy of parameter estimation. A fourth-order cumulant will be constructed, it can be expressed as

$$
\begin{aligned}
& C(m, i, n, q)=\operatorname{cum}\left(x_{m}, x_{i}^{*}, x_{n}^{*}, x_{q}\right) \\
& =\sum_{k=1}^{K} c_{4, s_{k}} e^{j\left\{[(m-i)-(n-q)] \mu_{k}+\left[\left(m^{2}-i^{2}\right)-\left(n^{2}-q^{2}\right)\right] \phi_{k}\right\}} \quad m, i, n, q \in[-M, M]
\end{aligned}
$$

where $c_{4, s k}=\operatorname{cum}\left\{s_{k}(t), s_{k}^{*}(t), s_{k}^{*}(t), s_{k}(t)\right\}, *$ stands for complex conjugate. In order to retain the azimuth and eliminate the range parameter in (13), both $\left(m^{2}-i^{2}\right)-\left(n^{2}-q^{2}\right)=0$ and $(m-i)-(n-q) \neq 0$, we assume $i=-m, q=-n[5]$. Then (13) becomes

$$
\begin{aligned}
C(m,-m, n,-n) & =\operatorname{cum}\left(x_{m}, x_{-m}, x_{n}, x_{-n}\right) \\
& =\sum_{k=1}^{K} c_{4, s_{k}} e^{j 2(m-n) \mu_{k}} \quad m, n \in[-M, M]
\end{aligned}
$$

Let $\bar{m}=m+M+1$ and $\bar{n}=n+M+1$, and thus $\bar{m}, \bar{n} \in[1,2 M+1]$, the $(\bar{m}, \bar{n})$ th element of special cumulant matrix $\mathbf{C}_{1}$ can be given by

$$
\begin{aligned}
& \mathbf{C}_{1}(\bar{m}, \bar{n})=\operatorname{cum}\left\{x_{\bar{m}-M-1}(k), x_{-\bar{m}+M+1}^{*}(k), x_{\bar{n}-M-1}^{*}(k), x_{-\bar{n}+M+1}(k)\right\} \\
& =\sum_{k=1}^{K} c_{4, s_{k}} e^{j 2(\bar{m}-M-1) \mu_{k}}\left(e^{j 2(\bar{n}-M-1) \mu_{k}}\right)^{*} \quad \bar{m}, \bar{n} \in[1,2 M+1] \\
& (2 M+1) \times(2 M+1) \text { matrix } \mathbf{C}_{1} \text { can be expressed as a compact matrix form } \\
& \mathbf{C}_{1}=\mathbf{A}_{\mu}(\theta) \mathbf{C}_{4 S} \mathbf{A}_{\mu}(\theta)^{H}
\end{aligned}
$$


where $\mathbf{A}_{\mu}(\theta)=\left[\mathbf{a}_{\mu}\left(\theta_{1}\right), \cdots, \mathbf{a}_{\mu}\left(\theta_{k}\right), \cdots, \mathbf{a}_{\mu}\left(\theta_{K}\right)\right]$ is a virtual steering matrix which is only relate to DOAs of sources, $\mathbf{a}_{\mu}\left(\theta_{k}\right)=\left[e^{-j 2 M \mu_{k}}, \cdots, 1, \cdots, e^{j 2 M \mu_{k}}\right]^{T}$ is the virtual steering vector, $\mathbf{C}_{4 s}=\operatorname{diag}\left[c_{4, s_{1}}, \cdots, c_{4, s_{k}}, \cdots, c_{4, s_{K}}\right], k=1, \cdots, K$. Then, we start to formulate the DOA estimation problem of the mixed sources through sparse representation. We sample the whole direction domain from a set $\Theta_{1}=\left\{\bar{\theta}_{1}, \cdots, \bar{\theta}_{N}\right\}$ with $N>>$. Assume the directions of actual sources only lie within the $N$ grids, the sparse signal representation of (16) is

$$
\mathbf{C}_{1}=\mathbf{A}_{\mu}\left(\Theta_{1}\right) \mathbf{S}_{S S}
$$

where $\mathbf{S}_{S S}=\mathbf{C}_{4, S_{N}} \mathbf{A}_{\mu}\left(\boldsymbol{\Theta}_{1}\right)^{H}$, it has the same sparseness as the signal, $\mathbf{A}_{\mu}\left(\boldsymbol{\Theta}_{1}\right)$ is the overcomplete basis, it can be expressed as

$$
\mathbf{A}_{\mu}\left(\boldsymbol{\Theta}_{1}\right)=\left[\begin{array}{ccccc}
e^{-j 2 \mu_{1} \times M} & e^{-j 2 \mu_{2} \times M} & \cdots & e^{-j 2 \mu_{n} \times M} & e^{-j 2 \mu_{N} \times M} \\
\vdots & \vdots & \vdots & \vdots & \vdots \\
1 & 1 & \cdots & 1 & 1 \\
\vdots & \vdots & \vdots & \vdots & \vdots \\
e^{j 2 \mu_{1} \times M} & e^{j 2 \mu_{2} \times M} & \cdots & e^{j 2 \mu_{n} \times M} & e^{j 2 \mu_{N} \times M}
\end{array}\right]
$$

In order to reduce the computation complexity, we extract the subspace only contains the signal information [11]. The signal value decomposition (SVD) from of (17) is

$$
\mathbf{C}_{1, S V}=\mathbf{A}_{\mu}\left(\Theta_{1}\right) \mathbf{S}_{S V}
$$

where $\mathbf{C}_{1}=\mathbf{U} \Lambda \mathbf{V}^{H}, \mathbf{C}_{1, S V}=\mathbf{C}_{1} \mathbf{V D} \mathbf{D}_{K}, \mathbf{S}_{S V}=\mathbf{S}_{S S} \mathbf{V D}_{K}, \mathbf{D}_{K}=\left[\mathbf{I}_{K}, \mathbf{0}_{K \times(2 M+1-K)}\right]^{T}$, $\mathbf{I}_{K}$ is a $K \times K$ identify matrix, $\mathbf{0}_{K \times(2 M+1-K)}$ denotes $K \times(2 M+1-K)$ zero matrix. Let $\left\|\mathbf{S}_{s v}(i,:)\right\|_{2}$ is the $\ell_{2}-$ norm of the $i$ th row of $\mathbf{S}_{S V}$, define $\mathbf{s}^{l 2}=\left[\left\|\mathbf{S}_{s v}(1,:)\right\|_{2}, \cdots,\left\|\mathbf{S}_{s v}(N,:)\right\|_{2}\right]^{T}$. The minimization $\ell_{1}$-norm of $\mathbf{s}^{l 2}$ is the objective function, the DOA estimation of the mixed sources can then be converted into the following optimization problem

$$
\begin{aligned}
& \min \sum_{i=1}^{N} w_{i}\left\|\mathbf{S}_{s v}(i,:)\right\|_{2} \\
& \text { s.t. } \mathbf{C}_{S V}=\mathbf{A}_{\mu}\left(\boldsymbol{\Theta}_{1}\right) \mathbf{S}_{S V}
\end{aligned}
$$

where $w_{i}$ is the weighted value, it can improve the performances of $\ell_{1}$-norm minimization which penalizes the larger elements in $\ell_{1}$-norm term more heavily than smaller ones [12]. $\mathbf{U}_{n}$ is the $(2 M+1) \times(2 M+1-K)$ noise-subspace matrix of $\mathbf{C}_{1}$, which is corresponding to the $2 M+1-K$ small signal value, $w_{i}$ can be expressed as

$$
w_{i}=\mathbf{a}_{\mu}\left(\theta_{i}\right)^{H} \mathbf{U}_{n} \mathbf{U}_{n}^{H} \mathbf{a}_{\mu}\left(\theta_{i}\right) .
$$

The optimization problem (20) can be efficiently solved by SOC software package of CVX [13]. 


\subsection{Range Estimation}

Once the DOAs $\hat{\theta}=\left[\hat{\theta}_{1}, \cdots, \hat{\theta}_{K}\right]$ are estimated, the next task is to estimate the range parameter relate to the DOAs. We construct another special fourth-order cumulant as follows

$$
\begin{aligned}
\mathbf{C}_{2}(\bar{m}, \bar{n}) & =\operatorname{cum}\left\{x_{m}^{*}(t), x_{m+1}(t), x_{-n}^{*}(t), x_{n}(t)\right\} \\
& =\sum_{k=1}^{K} C_{4, s_{k}} e^{j(2 m+1) \phi_{k}} e^{j(2 n+1) \mu_{k}}
\end{aligned}
$$

where $\bar{m}=m+M+1, \bar{n}=n+M+1, \quad m, n \in[-M, M-1]$. Then the $\bar{n}$ th column of the cumulant matrix $\mathbf{C}_{2}$ can be expressed as

$$
\mathbf{C}_{2}(\bar{n})=\sum_{k=1}^{K} \mathbf{a}_{\phi_{k}} C_{4, s_{k}} e^{j(2 n+1) \mu_{k}}=\mathbf{A}_{\phi} \mathbf{s}(\bar{n})
$$

where $\mathbf{a}_{\phi_{k}}$ is a $2 M$ vector

$$
\mathbf{a}_{\phi_{k}}=\left[e^{j(-2 M+1) \phi_{k}}, \cdots, e^{j(2 \times 0+1) \phi_{k}}, \cdots, e^{j(2 M-1) \phi_{K}}\right]^{T}
$$

$\mathbf{A}_{\phi}$ is a $2 M \times K$ matrix

$$
\mathbf{A}_{\phi}=\left[\mathbf{a}_{\phi_{1}}, \mathbf{a}_{\phi_{2}}, \cdots, \mathbf{a}_{\phi_{K}}\right]
$$

$\mathbf{s}(\bar{n})$ is a $K \times 1$ vector

$$
\mathbf{s}(\bar{n})=\left[C_{4, S_{1}} e^{j(2 n+1) \mu_{1}}, C_{4, S_{2}} e^{j(2 n+1) \mu_{2}}, \cdots, C_{4, S_{K}} e^{j(2 n+1) \mu_{K}}\right]^{T} .
$$

The cumulant matrix $\mathbf{C}_{2}$ could be regard as a virtual array output, with $\mathbf{s}(\bar{n})$ as the virtual signals and $\mathbf{a}_{\phi_{k}}$ as the steering vector, which only depend on $\phi_{k}$. Note that the near-field source is in the Fresnel region, and range $r$ is in the interval $\left[0.62\left(D^{3} / \lambda\right)^{1 / 2}, 2 D^{2} / \lambda\right]$ with $D$ representing the array aperture. We divide the whole range region of near-field source uniformly into $N_{1}$ girds, and a range set is given by $\mathbf{r}_{n}=\left[r_{n, 1}, \cdots, r_{n, N_{1}}\right]$. In order to distinguished mixed source, we define the range girds set for far-field sources with $\mathbf{r}_{f}=\left[r_{f, 1}, \cdots, r_{f, N_{2}}\right]$, the girds should be greater than the upper bound of the Fresnel region $2 D^{2} / \lambda$. For example, let $\mathbf{r}_{f}$ be $\left[4 D^{2} / \lambda, \cdots, 8 D^{2} / \lambda\right]$ with $D^{2} / \lambda$ interval in experiments. Then the sparse form of $\mathbf{C}_{2}$ can be expressed as

$$
\mathbf{C}_{2}=\mathbf{A}_{\phi, \boldsymbol{R}} \mathbf{X}
$$

where $\mathbf{X}$ of dimension $K\left(N_{1}+N_{2}\right) \times 2 M$ is the sparse of $\mathbf{S}$ with only $K$ non-zero rows, and $\mathbf{S}=[\mathbf{s}(1), \mathbf{s}(2), \cdots, \mathbf{s}(2 M)] . \quad \mathbf{A}_{\phi, \boldsymbol{R}} \quad$ of dimension 
$2 M \times K\left(N_{1}+N_{2}\right)$ is the overcomplete basis

$$
\begin{aligned}
\mathbf{A}_{\phi, \boldsymbol{R}} & =\left[\mathbf{A}_{\phi_{1}, r_{n}}, \mathbf{A}_{\phi_{1}, r_{f}}, \cdots, \mathbf{A}_{\phi_{k}, r_{n}}, \mathbf{A}_{\phi_{k}, r_{f}}, \cdots, \mathbf{A}_{\phi_{K}, r_{n}}, \mathbf{A}_{\phi_{K}, r_{f}}\right] \\
\mathbf{A}_{\phi_{k}, r_{n}} & =\left[\mathbf{a}\left(\phi_{k}, r_{n, 1}\right), \cdots, \mathbf{a}\left(\phi_{k}, r_{n, N_{1}}\right)\right] \\
\mathbf{A}_{\phi_{k}, r_{f}} & =\left[\mathbf{a}\left(\phi_{k}, r_{f, 1}\right), \cdots, \mathbf{a}\left(\phi_{k}, r_{f, N_{2}}\right)\right] .
\end{aligned}
$$

Similar to (19), the SVD version of the sparse representation (27) to reduce the computational complexity is given by

$$
\mathbf{C}_{2, S V}=\mathbf{A}_{\phi, \boldsymbol{R}} \mathbf{X}_{S V}
$$

Where $\quad \mathbf{C}_{2}=\mathbf{U}_{1} \boldsymbol{\Lambda}_{1} \mathbf{V}_{1}^{H} \quad, \quad \mathbf{C}_{2, S V}=\mathbf{C}_{2} \mathbf{V}_{1} \mathbf{H}_{K} \quad, \quad \mathbf{X}_{S V}=\mathbf{X} \mathbf{V}_{1} \mathbf{H}_{K} \quad$, $\mathbf{H}_{K}=\left[\mathbf{I}_{K}, \mathbf{0}_{K \times(2 M-K)}\right]^{T}, \quad \mathbf{I}_{K} \quad$ is a $K \times K$ identify matrix, $\mathbf{0}_{K \times(2 M-K)}$ denotes $K \times(2 M-K)$ zero matrix. Let $\left\|\mathbf{X}_{S V}(i,:)\right\|_{2}$ is the $\ell_{2}$ norm of the $i$ th row of $\mathbf{X}_{S V}$, define $\mathbf{s}_{r}^{l_{2}}=\left[\left\|\mathbf{X}_{S V}(1,:)\right\|_{2}, \cdots,\left\|\mathbf{X}_{S V}\left(K\left(N_{1}+N_{2}\right),:\right)\right\|_{2}\right]^{T}$. The minimization $\ell_{1}$-norm of $\mathbf{s}_{r}^{l_{2}}$ is the objective function, the range estimation can be realized by solving the following optimization problem

$$
\begin{aligned}
& \min \sum_{i=1}^{K \times\left(N_{1}+N_{2}\right)} w_{i}\left\|\mathbf{X}_{S V}(i,:)\right\|_{2} \\
& \text { s.t. } \mathbf{C}_{2, S V}=\mathbf{A}_{\phi, \boldsymbol{R}} \mathbf{X}_{S V}
\end{aligned}
$$

where

$$
w_{i}=\mathbf{A}_{\phi, \boldsymbol{R}}(:, i)^{H} \overline{\mathbf{U}}_{r, n} \overline{\mathbf{U}}_{r, n}^{H} \mathbf{A}_{\phi, \boldsymbol{R}}(:, i)
$$

where $\mathbf{A}_{\phi, \boldsymbol{R}}(:, i)$ is the $i$ th column of $\mathbf{A}_{\phi, \boldsymbol{R}}, \overline{\mathbf{U}}_{r, n}$ is the $(2 M) \times(2 M-K)$ noise-subspace matrix of $\mathbf{C}_{2}$, which is corresponding to the $2 M-K$ small signal value. The range parameter of sources can be obtained by solving the optimization problem(32). If the estimated range $r_{k}$ falls into the set $r_{n}$, the range parameter corresponds to the near-field source with DOA $\hat{\theta}_{k}$, otherwise, let $r_{k}$ be $\infty$ and the source lies in the far-field region.

\section{Discussion}

\subsection{Computational Complexity}

Regarding the computational complexity, we consider the major part. Define the search step of DOA with $\Delta \theta$ and the search step of range with $\Delta r$. The major computation of the algorithm [5] is to constructs one $(2 M+1) \times(2 M+1)$ and one $(4 M+1) \times(4 M+1)$ cumulant matrix, to perform EVD of the two matrix, to execute MUSIC spectral search corresponding to the DOA estimation. Therefore, the computational complexity is O\{ $\left.9(2 M+1)^{2} T+9(4 M+1)^{2} T+4 / 3(2 M+1)^{3}+4 / 3(4 M+1)^{3}+180(2 M+1)^{2} / \Delta \theta\right\}$. The 
major computation of the algorithm[7] is to form one $(2 M+1) \times(2 M+1)$ and one $(M+2) \times(M+2)$ matrix, to perform eigen-decompositions of the two matrix, to execute MUSIC spectral search corresponding to the DOA and range estimation. Therefore, the computational burden of algorithm [7] is $\mathrm{O}\left\{(2 M+1)^{2} T+(M+2)^{2} T+4 / 3(2 M+1)^{3}+\right.$

$$
\left.4 / 3(M+2)^{3}+2 \pi / \Delta \theta(2 M+1)^{2}+K\left(2 D^{2} / \lambda-0.62\left(D^{3} / \lambda\right)\right)(2 M+1)^{2} / \Delta r \quad\right\} .
$$

However, for the proposed algorithm, we construct one $(2 M+1) \times(2 M+1)$ and one $2 M \times 2 M$ cumulant matrix, and implements their eigen-decomposition, the corresponding sparse signal reconstruction process requires $\mathrm{O}\left\{K^{3} N^{3}+K^{6}\left(N_{1}+N_{2}\right)^{3}\right\}$. Therefore, the complexity of the proposed method is $\mathrm{O}\left\{9(2 M+1)^{2} T+9(2 M)^{2} T+4 / 3(2 M+1)^{2}+4 / 3(2 M)^{2}+K^{3} N^{3}+\right.$

$$
\left.K^{6}\left(N_{1}+N_{2}\right)^{3}\right\} \text {. }
$$

From the analysis above, it can be seen that the computational complexity of the proposed method is much lower than that of [5]. Note that the main complexity of the algorithm [7] is in calculating second-order statistics and its eigenvalue decomposition, which is lower than the proposed algorithm. While the proposed algorithm used the cumulant, it alleviates the aperture loss. It is important note that the proposed algorithm can provide an improved DOA and range estimation.

\subsection{Array Aperture}

With an uniform linear array of $2 M+1$ sensors, the algorithm of [5] can construct $(2 M+1) \times(2 M+1)$ dimensional matrix. It needs at least one eigenvector of the constructed matrix to span the noise subspace. Thus, the algorithm [5] can localize $2 M$ sources at most. The algorithm [7] can only localize $M$ sources because it has half aperture loss. The proposed algorithm has the same array aperture with the algorithm [5], then it has much better performance than the algorithm [7] at array aperture.

\subsection{Estimation Accuracy}

On the one hand, the proposed algorithm has the same array aperture with the algorithm [5] and has larger array aperture than the algorithm [7]; one the other hand, the algorithm [5] and algorithm [7] estimation the DOAs parameters of sources by means of MUSIC spectral search, whereas the proposed algorithm used the weighted $\ell_{1}$-norm algorithm for sparse signal reconstruction, which can promote higher resolution and estimation. Therefore, the proposed algorithm is expected to have better estimation performance of both DOAs and range parameters than the compared algorithm.

\section{Simulations}

In this section, the proposed algorithm will compared with high-order MUSIC algorithm [5] and second-order statistic (SOS)-based algorithm [7] to demonstrate the choiceness performance. In the following simulations, a 7-element symmetric uniform liner array with element spacing $d=1 / 4 \lambda$ is considered. The root-mean-square error (RMSE) that indicates the performance of the proposed algorithm is obtained by 400 independent Monte Carlo simulations.

In the first experiments, we compared the RMSE of the DOA and range estimation 
versus SNR, whose curves are plotted in Figure 1. Two sources located at $\left\{\theta_{1}=-13^{\circ}, r_{1}=2 \lambda\right\}$ and $\left\{\theta_{2}=21^{\circ}, r_{2}=\infty\right\}$ are considered. The number of snapshots is fixed at 600 , while the SNR is varied from $-5 \mathrm{~dB}$ to $13 \mathrm{~dB}$ in steps of $2 \mathrm{~dB}$. From the simulation results, we observed that the estimation performance of the algorithm proposed is better than the compared methods. Algorithm [7] has the worst DOA estimation accuracy because of it has half aperture loss. The proposed algorithm has the same array aperture with the algorithm[5], whereas the algorithm [5] estimates the DOA parameters of sources by means of MUSIC spectral search, the proposed algorithm uses the sparse signal reconstruction, which can promote better DOA and range estimation. In addition, it is obvious that the RMSE decreases monotonically with the number of SNR. As for the range estimation, the proposed algorithm is still superior to algorithm [5] and algorithm [7], which is mainly a result of the propagation error from the previous DOA estimation stage.
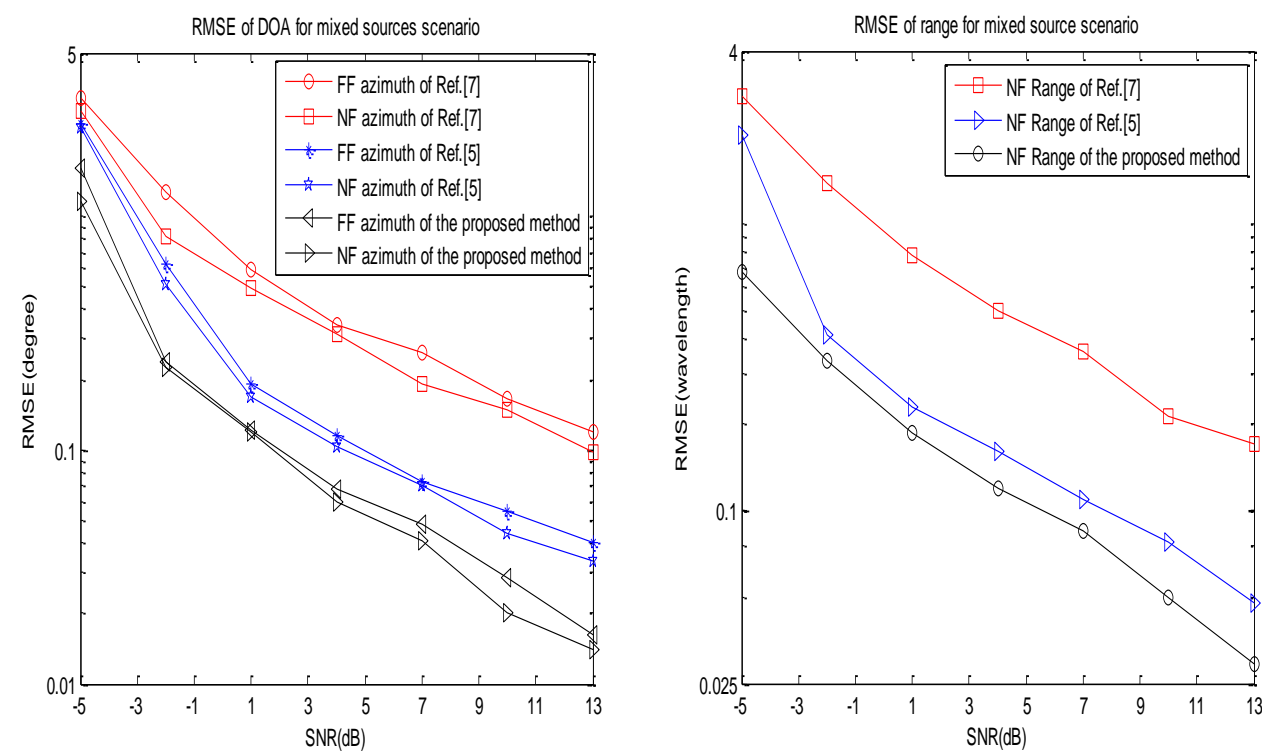

\section{Figure 1. The RMSE of Azimuth DOA Estimation and Range Estimation versus SNR}

In the second experiment, we compared the RMSE of the DOA and range estimation versus snapshot, whose curves are plotted in Figure 2. The simulation condition is similar to the first experiment except that the SNR is fixed at $7 \mathrm{~dB}$, and the snapshot number is varied from 100 to 1300 in 200 steps. We can see that the RMSE of the DOA and range decrease monotonically as the snapshot number increases. This is due to the fact that a larger sampling number will produce better estimation of the cumulant matrices and the covariance matrices for stationary data. In addition, it is obvious that the performance of the proposed algorithm is the best, and the performance of the method in [7] is the worst one. This is because the algorithm [7] suffer a half aperture loss, the algorithm proposed and the algorithm [7] constructed the cumulant matrices to alleviate the aperture loss. 

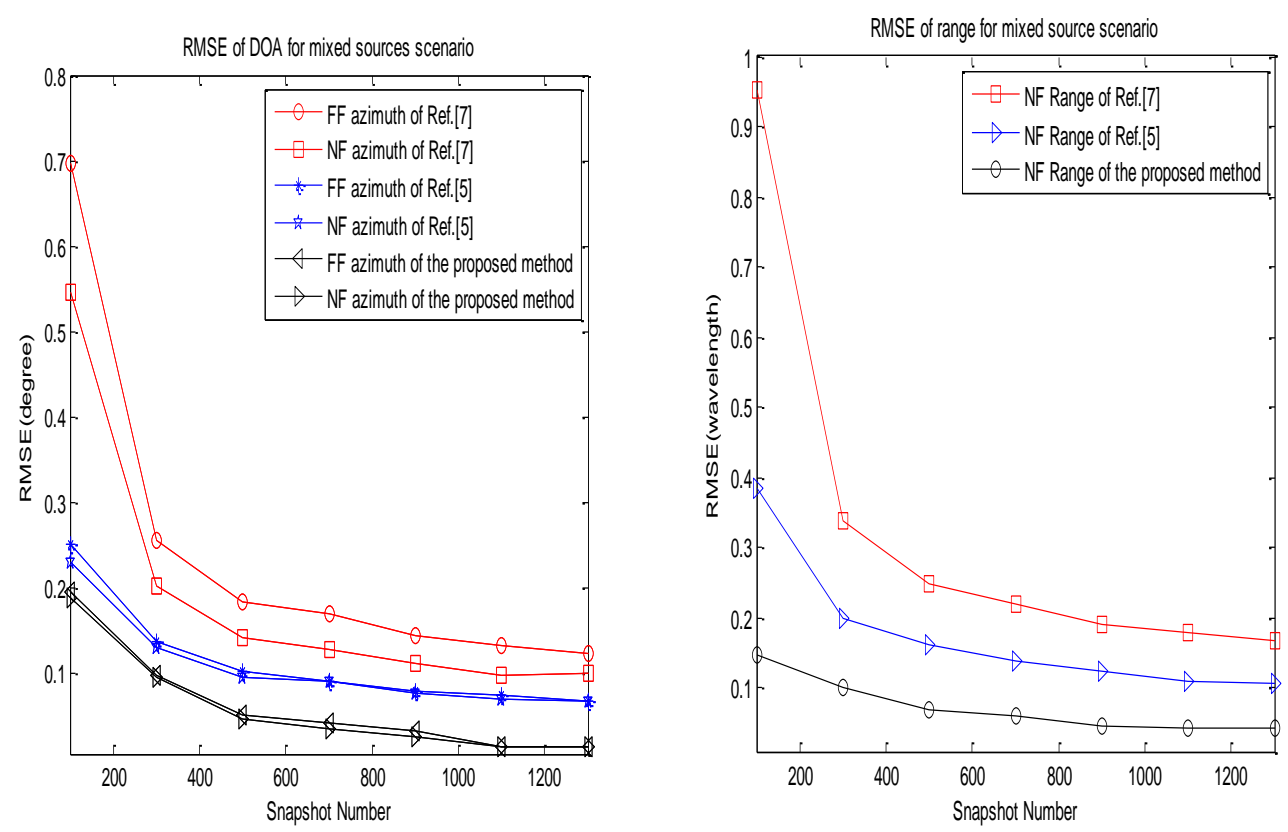

\section{Figure 2. The RMSE of Azimuth DOA Estimation and Range Estimation versus Snapshot}

In the third experiment, we examine the resolution ability of the proposed algorithm and the algorithm in [5] and [7]. The resolution probability is the ratio between the number of successful estimation and the number of total trials. when $\left|\hat{\theta}_{k}-\theta_{k}\right|<\Delta / 2$, estimation is considered to be successful[14], where $\hat{\theta}_{k}$ is the estimation value, $\theta_{k}$ is the real value, $\Delta$ is the angle-interval of two sources. We will do experiments for two closely space sources including two scenarios: (1) two far-field sources located at $\left\{\theta_{1}=16^{\circ}, r_{1}=\infty\right\}$ and $\left\{\theta_{2}=20, r_{2}=\infty\right\}$, the angle-interval is $4^{\circ}$; (2) two near-field sources located at $\left\{\theta_{1}=14^{\circ}, r_{1}=2 \lambda\right\}$ and $\left\{\theta_{2}=10^{\circ}, r_{2}=3 \lambda\right\}$, the angle-interval is $4^{\circ}$. The resolution probabilities versus SNR for $N=700$ snapshots are provided in Figure 3 . The results show that the proposed algorithm has better resolution ability than the compared method. The algorithm [7] has the worst resolution ability because it suffer a half aperture loss, while the proposed algorithm and algorithm [5] avoid the aperture loss. In addition, the proposed algorithm estimate the DOAs of all sources by using weighted $\ell_{1}$-norm algorithm, compared with the subspace technique which used in [5], it can promotes the resolution ability. 

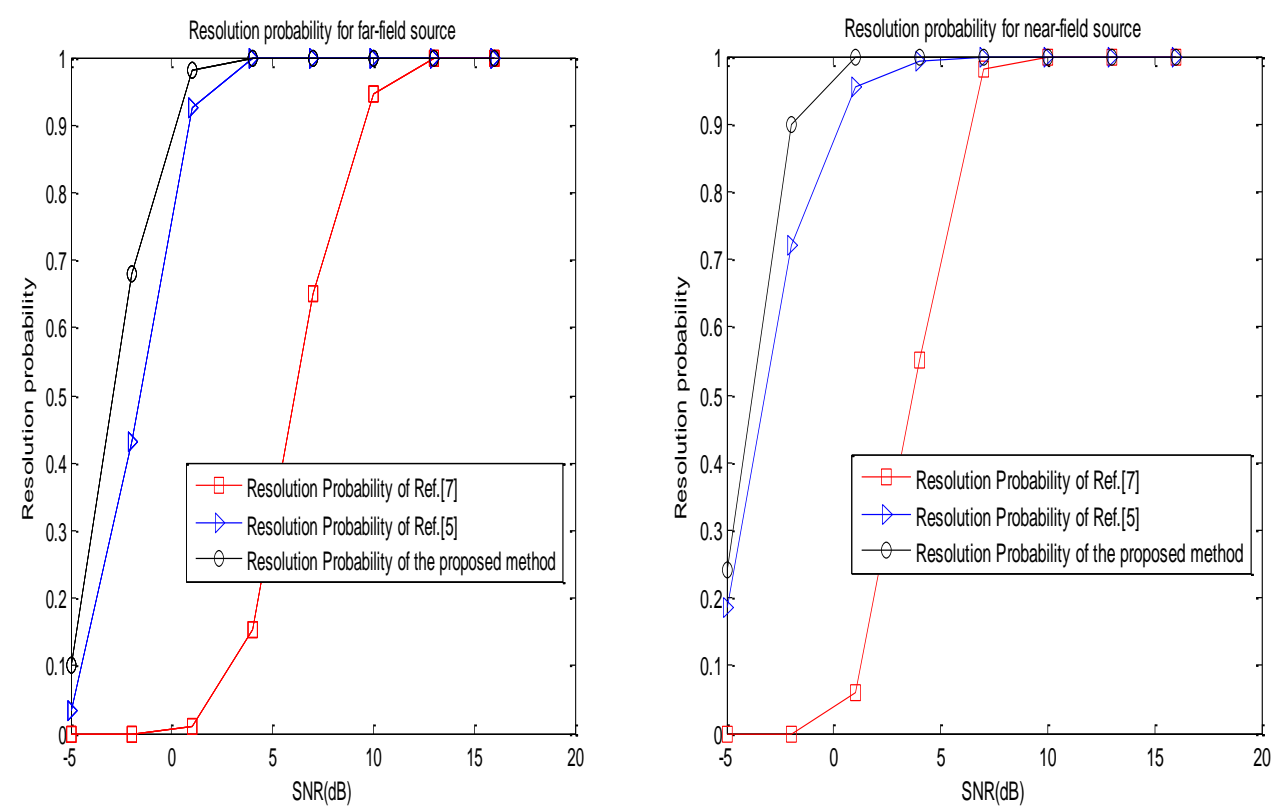

Figure 3. Resolution Probability versus SNR for Closing Spaced Sources

In the last simulation, the computational cost required by the proposed algorithm is compared with the algorithm [5] and algorithm [7]. The number of sensors $2 M+1$ is set to 7. Further we define $\Delta \theta=0.1^{\circ}, \Delta r=0.01 \lambda, K=2$. Figure4 shows the computational complexity of these algorithms as a function of the number of snapshots. It can be seen clearly from Figure 4 that the computational complexity of the proposed method is much lower than that of [5]. Note that the main complexity of the method addressed in [7] is in calculating the array covariance matrix and its eigenvalue decomposition, which is lower than the proposed algorithm. However, the proposed algorithm has better estimation accuracy, it can be regard as a good compromise between the estimation accuracy and the computational complexity.

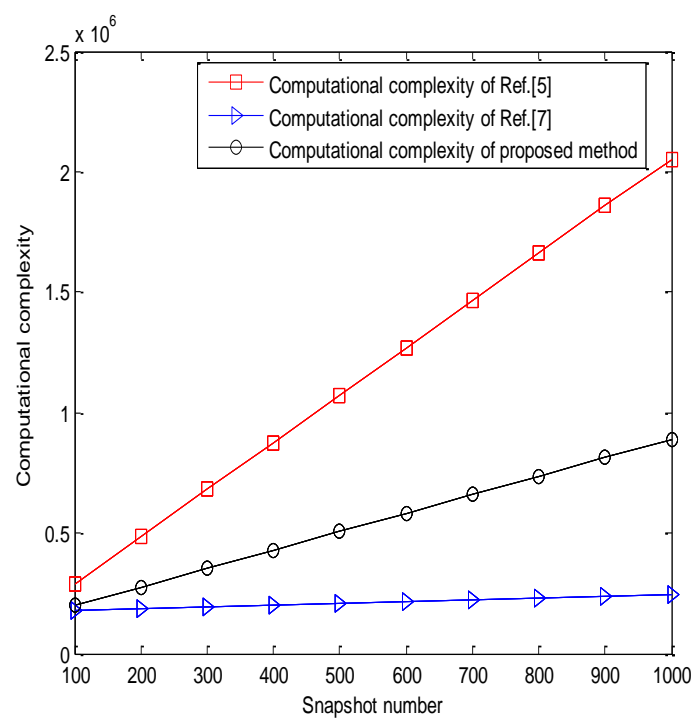

Figure 4. Computational Complexity of These Three Methods versus Snapshot Number 


\section{Conclusion}

In this paper, we proposed a new mixed sources localization method using sparse representation of the cumulant matrix. Two special cumulant matrix are constructed, the first one only contains the DOAs, the second one contains both DOAs and the range parameters. And then, we construct two overcomplete bases to reconstruct the fourth-order cumulant matrix to estimate the two parameters separately. When the sparse representation model is solved by using weighted $\ell_{1}$-norm algorithm, it's unnecessary to select the regularization parameter which balances reconstruction residual with the sparsity of solution. Moreover, the proposed algorithm avoids parameter match and two-dimensional search. The simulation results demonstrate the higher estimation accuracy and resolution probability than the compared method.

Although the algorithm proposed has good performance, it still has some limitations. First, in order to reduce the computation complexity, we implement the SVD on the constructed cumulant matrix, thus it must have a priori knowledge of the number of source. Second, base on the virtual cross-correlation computer $\left(\mathrm{VC}^{3}\right)$ theory [15], the estimation accuracy increases as the number of virtual sensors increases, the proposed algorithm only avoid aperture loss. To put the proposed algorithm into further applications, several steps of the future work can be done as follows:

1. To avoid having a priori knowledge of the number of sources, we can use reweighted $\ell_{1}$-norm minimisation for sparse signal recovery without implementing the SVD on the constructed cumulant matrix.

2. To extend the array aperture, we can use the sparse uniform linear array or co-prime array instead of uniform linear array.

\section{Acknowledgement}

This work was supported in part by the National Natural Science Foundation of China (Grant Nos. 61461012, 61371186), Guangxi Key Lab of Wireless Wideband Communication \& Signal Processing (Grant No. GXKL06160110), Center for Collaborative Innovation in the Technology of IOT and the Industrialization (Grant No. WLW20060205), Innovation Project of GUET Graduate Education (Grant Nos. GDYCSZ201455, 2016YJCX88).

\section{References}

[1] Y. Ma, B. X. Chen, M. L. Yang and Y. Wang, "A Novel ESPRIT-Based Algorithm for DOA Estimation with Distributed Subarray Antenna”, Circuits Systems \& Signal Processing, vol. 34, no. 9, (2015), pp. 2951-2972.

[2] Q. H. Liu, N. L. Jin and S. Ouyang, "Wideband DOA Estimation with Interpolated Focusing KR Product Matrix", International Journal of Signal Processing Image Processing \& Pattern Recognition, vol. 8, no. 3, (2015), pp.201-210.

[3] Y. D. Huang and M. Barkat, "Near-field multiple source localization by passive sensor array", IEEE Transactions on Antennas \& Propagation, vol. 39, no. 7, (1991), pp. 968-975.

[4] N. Yuen and B. Friedlander, "Performance analysis of higher order ESPRIT for localization of near-field sources”, IEEE Transactions on Signal Processing, vol.46, no.3, (1998), pp.709-719.

[5] J. Liang and D. Liu, "Passive localization of mixed near-field and far-field sources using two-stage MUSIC algorithm", IEEE Transactions on Signal Processing, vol. 58, no. 1, (2010), pp. 108-120.

[6] G. H. Liu and X. Y. Sun, "Efficient Method of Passive Localization for Mixed Far-Field and Near-Field Sources", IEEE Antennas \& Wireless Propagation Letters, vol. 12, no. 4, (2013), pp. 902-905.

[7] J. He, M. N. S. Swamy and M. O. Ahmad, "Efficient Application of MUSIC Algorithm Under the Coexistence of Far-Field and Near-Field Sources”, IEEE Transactions on Signal Processing, vol. 60, no. 4, (2012), pp. 2066-2070.

[8] D. Malioutov, M. Cetin and A. S. Willsky, "A sparse signal reconstruction perspective for source localization with sensor arrays", IEEE Transactions on Signal Processing, vol. 53, no. 8, (2010), pp. 3010-3022.

[9] B. Wang, J. J. Liu and X. Y. Sun, "Mixed Sources Localization Based on Sparse Signal Reconstruction", 
IEEE Signal Processing Letters, vol. 19, no. 8, (2012), pp. 487-490.

[10] J. Xie, H. H. Tao, X. Rao and J. Su, "Localization of mixed far-field and near-field sources under unknown mutual coupling", Digital Signal Processing, vol. 50, no. C, (2015), pp. 229-239.

[11] Y. Tian and X. Y. Sun, "Passive localization of mixed sources jointly using MUSIC and sparse signal reconstruction", AEU - International Journal of Electronics and Communications, vol. 68, no. 6, (2014), pp. 534-539.

[12] C. D. Zheng, G. Li Y. Liu and X. Q. Wang, "Subspace weighted $\ell$ 2,1 minimization for sparse signal recovery", Journal on Advances in Signal Processing, vol. 98, no.1, (2012), pp. 1-11.

[13] Grant M and Boyd S, "CVX: MATLAB software for disciplined convex programming”, June 10, 2015, available at: http://www.cvx.com/cvx.

[14] B. Wang, Y. P. Zhao and J. J. Liu, "Mixed-Order MUSIC Algorithm for Localization of Far-Field and Near-Field Sources", IEEE Signal Processing Letters, vol. 20, no. 4, (2013), pp. 311-314.

[15] M.C. Dogan and J. M. Mendel, "Applications of cumulants to array processing-Part I: Aperture extension and array calibration", IEEE Trans. Signal Process, vol. 43, no.5, (1995), pp. 1200-1216. 